\title{
Throughput Maximization in Cognitive Radio Network Under Constant Fading using Hierarchical Neural System
}

\author{
Neetu Goyal, Sanjay Mathur
}

\begin{abstract}
Cognitive radios (CRs) predominantly reuse the spectrum holes to proficiently utilize the scarcely available radio spectrum. In the CRs, the throughput limitation is a major difficulty among the key limitations such as energy consumption, processing resources, cost, and quality of service limitations, that affects a wide range of telecommunication applications nowadays. Moreover, attaining high throughput will overcome the bottleneck of CRs applications. To overcome this emerging throughput limitation issue in the CRs, this paper proposes the best channel prediction algorithm using Multilayer Feed forward Neural Network (MFNN) which tackles the throughput limitations.
\end{abstract}

Keywords: Cognitive Radios, Constant Fading, Multilayer Feed Forward Neural Network, Channel Prediction, Throughput.

\section{INTRODUCTION}

According to a forecast, global mobile data traffic will increase seven-fold between the year 2017 and year 2022 [1]. An exponential increase in demand for wireless spectrum has put it into a 'precious and scarce' category resource. Due to constrain of a fixed spectrum allocation policy, it is not possible to overcome the scarcity and support exponential upsurge in demand. However, cognitive radio (CR) is looked upon as an effective solution to this problem with an increasing amount of interest over the past decade. In cognitive radio system unlicensed user called as secondary user (SU) is allowed to access the spectrum which is preallocated to licensed or primary user (PU) under the condition of protecting the quality of service (QoS) of the licensed (primary) networks [2-4]. In cognitive radio, an SU can access the licensed spectrum majorly in two ways: (i) the opportunistic spectrum access (OSA) where a secondary user accesses a frequency band only when it is detected to be idle [3] and (ii) the spectrum sharing (SS), where the secondary users coexist with the primary users conditionally protecting the latter from harmful interference [5].A dynamic spectrum management framework (DSMF) is required for realizing efficient spectrum utilization by CR technology. It consists of spectrum sensing, spectrum decision, spectrum sharing, and spectrum mobility [4]. In this paper we focus on spectrum decision factor of DSMF.

Revised Manuscript Received on February 05, 2020.

* Correspondence Author

Neetu Goyal, Pursuing Ph.D., Govind Ballabh Pant University of Agriculture and Technology, Pantnagar, India.

Sanjay Mathur, Professor in the Department of Electronics and Communication Engineering, Govind Ballabh Pant University of Agriculture and Technology, Pantnagar.

(C) The Authors. Published by Blue Eyes Intelligence Engineering and Sciences Publication (BEIESP). This is an open access article under the CC BY-NC-ND license (http://creativecommons.org/licenses/by-nc-nd/4.0/)
Spectrum decision is explained as the selection of best out of multiple available spectrum bands for SUs to improve the utilization. Spectrum decision consists of three main steps: Spectrum characterization, spectrum selection, and CR reconfiguration [4]. A detailed description of spectrum decision framework is presented in [6] and in [7] spectrum sensing combined with prediction was proposed using dual optimal theory to reduce the interference with PUs and enhance the spectrum utilization. A comprehensive survey on spectrum decision in CR networks is presented in [8]. A cognitive source node typically completes its transmission, to the destination in two essential phases: 1) a spectrum sensing phase, wherein the cognitive source detects a spectrum hole with fixed time duration (referred to as spectrum sensing over-head) and 2) a data transmission phase, wherein data is transmitted to the destination through the detected spectrum hole as shown in Fig.1. The two phases have been studied individually in terms of different detection [9] - [11] or different transmission techniques. It is to be noted that the spectrum hole detection and data transmission phases have to be designed and optimized together since they could affect each other's performance. It is clear that a trade-off between detection performance and frame efficiency exists in CR systems. An available spectrum hole would get wasted if the cognitive source is unable to detect the hole within certain time duration which would affect the spectrum hole utilization efficiency, on the other hand increasing the detection phase improves the detection probability of spectrum holes, but reduces transmission time of SU remaining for the data transmission. The performance metrics of the spectrum sensing is similar to any detection problem characterized by detection probability, $\mathrm{P}_{\mathrm{d}}$ and probability of false alarm, $\mathrm{P}_{\mathrm{fa}}$. The values of $\mathrm{P}_{\mathrm{d}}$ and $\mathrm{P}_{\mathrm{fa}}$ must be high and low respectively for reliable performance. To obtain this, the sensing time has to be significantly high, but at a cost of lower throughput due to sensing-throughput trade-off [12-13]. To maximize throughput while maintaining the sensing reliability, researchers are proposing various techniques. A new direction in research is being observed where predicting the spectrum hole in next frame to be transmitted by SU is being identified as an effective way of maximizing the throughput without sacrificing the transmission time. This approach suggests that prediction based sensing and data transmission may go in parallel and throughput will depend on prediction accuracy only. In order to maximize throughput and sensing reliability [14] modified the sensing-throughput trade-off using improved frame structure by performing data transmission and sensing at the same time. Throughput enhancement by using same frame structure as in [14] but with reduced data loss rate by dividing the transmission time into small segments is proposed in [15].

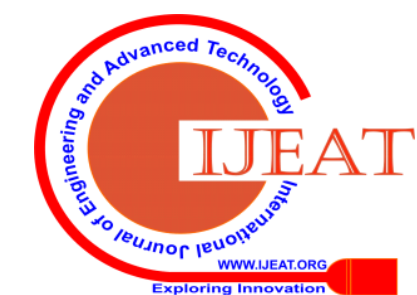


In high traffic CRN SU's low throughput problem is solved in [16] by redesigning the frame structure with prediction phase. Here the selection of channel for sensing from among all idle predicted channels is random which may cause as sense and stuck problem. To solve this problem a method for improved selection of sensing channel that relies on the probability of idle prediction of channel is proposed in [17]. In both the pre-existing approaches [16] and [17] prediction is done for the channel state only not for channel selection if multiple channels are coexisting in the CRN and may be available to SU. In this paper, prediction is done for best channel to maximize throughput.

\section{SYSTEM DESCRIPTION}

Throughput in cognitive radio network depends on the data transmission time available for secondary user. If this value is more, throughput is more. Conventional cognitive transmission frame is shown below:

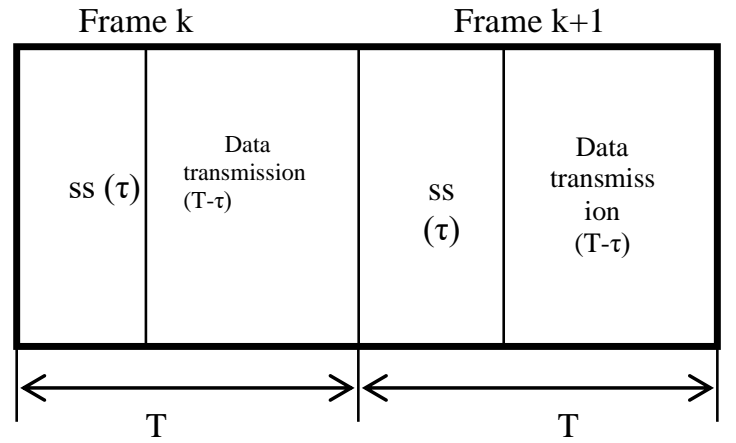

Figure 1: Conventional Cognitive Transmission Frame

For a frame $\mathrm{k}, \mathrm{T}(\mathrm{ms})$ is the total time in which $\tau$ is spectrum sensing (ss) time and (T- $\tau$ ) is for data transmission time. If $\tau$ is less then only data transmission time will be more. But because the sensing time is essential for detecting primary user and it is directly proportional to the probability of detection $\left(\mathrm{P}_{\mathrm{d}}\right)$, Low $\mathrm{P}_{\mathrm{d}}$ fails to detect primary user and collision chances will be more. Hence there is a trade-off occur [12] in sensing time and throughput which is explained in literature. In our analysis for a frame $\mathrm{k}+1$ whole time $\mathrm{T}$ is utilized for transmission as we have done parallel processing and not doing sensing and in frame $\mathrm{k}$ best channel is predicted for frame $k+1$. By using this frame structure and prediction throughput gets maximized.
Frame k

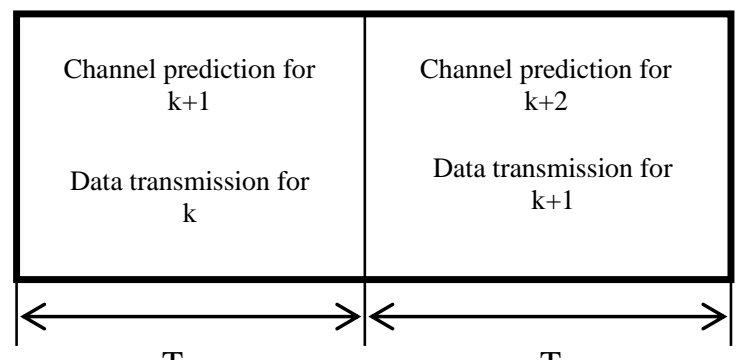

$\mathrm{T}$
Figure 2: Proposed Frame Structure

In constant fading, fading is same for all 3000 frames but different for 8 channels. Input data set $F$ is generated by simulating above algorithm in MATLAB for predicting best channel. We apply obtained input data in neural network and in order to speed up learning process input data set is normalized to make full data set into the interval $[0,1]$. We also introduce memory in normalized input data set such that $x(n)=F(n-k)$, where $k=0,1,2$. Hence for our analysis we have 8 inputs for present frame $k, 8$ inputs from past frame $\mathrm{k}-1$ and another 8 inputs for past frame k-2. This build input data set of total 24 values. The output is to be the futuristic best channel indexing. In the hierarchical structure shown in fig.3 apply input data set to NN1, it gives channel index ranging from 1-8 as an output. We further classify the input dataset according to the output into odd and even. The input data set of the odd output values $(1,3,5,7)$ are further processed in NN11 while the input data set of the even output values $(2,4,6,8)$ are processed in NN12. Further the dataset of output values of NN11 are divided into two parts $-(1,5)$ and $(3,7)$ and are further processed in NN111 $(1,5)$ and NN112 $(3,7)$. Similarly dataset of output values of NN12 are divided into two parts - $(2,6)$ and $(4,8)$ and are further processed in NN121 $(2,6)$ and NN122 $(4,8)$. Finally we get the output values through NN111, NN112, NN121 and NN122. From these results predicted channel is to be found and through simulations throughput is generated.

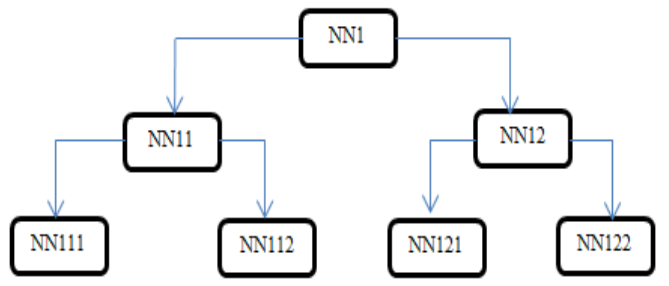

Figure 3: Hierarchical Neural System

The spectrum occupancy in most licensed user systems encountered in reality is non-deterministic in nature. Hence, it is appropriate to model such traffic characteristics using nonlinear adaptive schemes. Neural networks are nonlinear parametric models which create a mapping function between the input and output data. Multilayer feed forward Neural Network (MFNN) and Hidden Markov Model (HMM) based prediction schemes are used in various kind of scenario. We use MFNN because it has many advantage over HMM scheme such as MFNN trained only once, requires less memory space, strong learning ability, requires fewer assumed conditions so MFNN is more effective in practical cases. Hence we use MFNN for prediction.

\section{RESULT AND DISCUSSION}

For simulation we consider 8 numbers of channels, number of SU is $1, \mathrm{SNR}$ is $10 \mathrm{~dB}$. As defined in IEEE 802.22 the frame transmission duration is $T(10 \mathrm{~ms})$ and each time slot must have duration of $2 T$ to ensure the successful exchange of information. The duration of each time slot in the simulation is considered to be $20 \mathrm{~ms}$ and packet data rate to be 22.69Mbps based on the existing standard [15]. In our simulation, throughput is defined as the number of data packets successfully transmitted per unit second. Thus, throughput is measured as

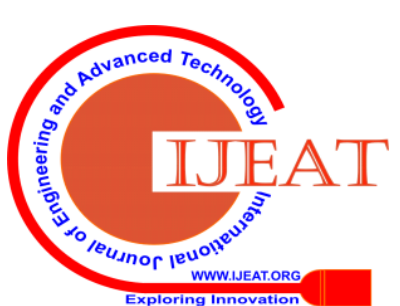




\section{(Number of bits transmitted)}

elapstime in seconds

We generate 2957 input data samples in which 2000 samples are used for training and 857 samples are used for testing the neural network. Again we take 100 samples and we see from the graph that the throughput for proposed work is significantly better than the throughput when the channel for SU chosen randomly. The upper bound for this graph is taken as the throughput when the channel for SU is always best. In our throughput it is possible when the channel chosen is best but it is not available for future state hence in this case it chooses second optimal best channel for communication, hence throughput decreases. But from the graph it is clear that average throughput for proposed work is lies between upper bound and the worst case (i.e. channel chosen randomly for communication).

\section{Hierarchical Neural System for channel prediction}

Neural network architecture with the details given in the table I was used to train the network with 2000 frames of all 8 channels with the current channel availability vector of dimension 8 corresponding to two immediate past channel availability vectors thus making an input of vector dimension of 24 . The training process was to train the network with the best available channel in the next frame.

Table I: Neural network architecture details and performance during training phase in constant fading

\begin{tabular}{|c|c|c|c|c|c|c|}
\hline $\begin{array}{c}\text { Neural } \\
\text { Network }\end{array}$ & Structure & LR & MR & Iterations & MSE & $\begin{array}{c}\text { Train } \\
\text { results } \\
\text { (in \%) }\end{array}$ \\
\hline NN1 & $24-24-1$ & 0.001 & 0.5 & 40000 & 0.020096 & 96.10 \\
\hline NN11 & $24-24-1$ & 0.7 & 0.5 & 5000 & 0.007254 & 98.54 \\
\hline NN12 & $24-24-1$ & 0.7 & 0.5 & 5000 & 0.005637 & 98.87 \\
\hline NN111 & $24-24-1$ & 0.7 & 0.5 & 5000 & 0.008630 & 98.17 \\
\hline NN112 & $24-24-1$ & 0.7 & 0.5 & 5000 & 0.002891 & 99.40 \\
\hline NN121 & $24-24-1$ & 0.7 & 0.5 & 5000 & 0.003732 & 99.25 \\
\hline NN122 & $24-24-1$ & 0.7 & 0.5 & 5000 & 0.006161 & 98.76 \\
\hline
\end{tabular}

All the neural networks converge to sufficiently low MSE and high accuracy in binary classification of channel vectors.

The validation of model using the 857 frames were not included in training, was done and the results obtained are as shown in table II.

Table II: Neural Network Architecture Details and Performance during Validation Phase

\begin{tabular}{|c|c|c|}
\hline Neural Network & Structure & $\begin{array}{c}\text { Validation results } \\
\text { (in\%) }\end{array}$ \\
\hline NN1 & $24-24-1$ & 47.14 \\
\hline NN11 & $24-24-1$ & 79.86 \\
\hline NN12 & $24-24-1$ & 69 \\
\hline NN111 & $24-24-1$ & 69.56 \\
\hline NN112 & $24-24-1$ & 63.75 \\
\hline NN121 & $24-24-1$ & 55.74 \\
\hline NN122 & $24-24-1$ & 78.09 \\
\hline
\end{tabular}

The validation criterion was set that on an average the validation accuracy should be much above at least $50 \%$ thus crossing the threshold of $50 \%$ accuracy expected from a random selection system. The overall average of testing results during validation is $66 \%$ which shows that prediction surpasses the pure chance expectation.

Once the network is trained and validated the system was fed with the input data for 100 frames to predict the best available channel and the throughput corresponding to the predicted channel was plotted.

Fig.4 shows the plots for throughput obtained corresponding to the best available channel and the channel predicted by the proposed system.It is found that the system is able to follow the trend of best available channel in a good conformity. It is found that the throughput for actually best available channel case is 0.7746 whereas the proposed model yielded a throughput of 0.7289 both averaged over 100 frames. Thus the system is able to achieve $94.10 \%$ upper bound when compared to the best available channel throughput normalized to $100 \%$.

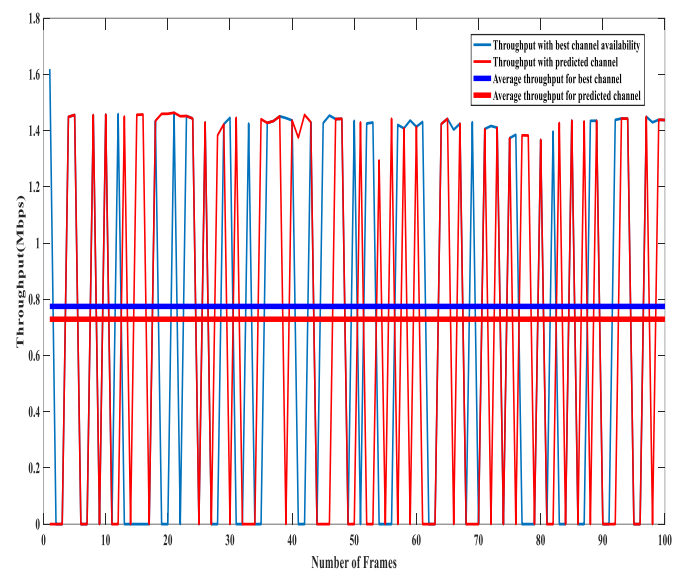

Figure 4: Throughput versus number of frames

In [16] a frame structure is proposed, in which the SU predicts the state of the channel (active or idle) before sensing and senses only the idle predicted channels. Due to the random sensing of channels in the CRNs, the probability of a channel to be sensed as active becomes significantly high, resulting in high data loss due to which the effective throughput of the network decreases significantly.

In [17] an approach in which selection relies on the highest probability of the channel being predicted idle is proposed.

Hence in both the pre-existing approaches prediction is done for the channel state only not for channel selection if multiple channels are coexisting in the CRN and may be available to SU. In this paper, prediction is done for best channel to maximize throughput.

\section{CONCLUSION}

This paper aims to maximize the throughput for secondary user in cognitive radio. Spectrum decision plays an important role towards this goal. In this paper, a framework for spectrum decision based on best channel prediction using neural network has been proposed. The simulation results reveal that the significant improvements in the throughput of the SUs have been achieved for proposed approach and the proposed spectrum decision framework is effective. In future, authors will extend this work for different kind of fading like cyclostationary and fast fading environment. 


\section{REFERENCES}

1. Cisco Visual Networking Index: Global Mobile Data Traffic Forecast Update, 2017-2022, pp. 1-36, white paper , February 2019.

2. J. Mitola and G. Q. Maguire, "Cognitive radio: Making software radios more personal”, IEEE Pers. Commun., vol. 6, no. 4, pp. 13-18, 1999.

3. S. Haykin, "Cognitive radio: brain-empowered wireless communications”, IEEE J. Sel. Areas Communication, vol. 23, no. 2, pp. 201-220, 2005.

4. I. F. Akyildiz, W.Y. Lee., M. C. Vuran and S. Mohanty, "NeXt generation/dynamic spectrum access/cognitive radio wireless networks: a survey", Computer Network, vol. 50, no. 13, pp. $2127-$ 2159, 2006.

5. A. Ghasemi and E. S. Sousa, "Fundamental limits of spectrumsharing in fading environments", IEEE Transactions on Wireless Communications, vol. 6, no. 2, pp. 649-658, 2007.

6. W. Y. Lee and I. F. Akyildiz, "A spectrum decision framework for cognitive radio networks", IEEE Transactions on Mobile Computing, vol. 10, no. 2, pp. 161-174, 2011.

7. Z. Zhao, L. Wang, G. Ding and S. Wei, "Enhanced spectrum decision based on the combination of sensing and prediction", In Proceedings of Cross Strait Quad-Regional Radio Science and Wireless Technology Conference (CSQRWC), pp. 985-989, 2011.

8. M. T. Masonta, M. Mzyece and N. Ntlatlapa, "Spectrum Decision in Cognitive Radio Networks: A Survey", IEEE Communications Surveys \& Tutorials, vol. 15, no. 3, pp. 1088-1107, 2013.

9. T. Yucek and H. Arslan, "A survey of spectrum sensing algorithms for cognitive radio applications", IEEE Communication Surveys \& Tutorials, vol. 11, no. 1, pp. 116-130, 2009.

10. H. Sun, A. Nallanathan, C. X. Wang and Y. Chen, "Wideband spectrum sensing for cognitive radio networks: a survey", IEEE Wireless Communications, vol. 20, no. 2, pp. 74-81, 2013.

11. S. K. Sharma et al., "Cognitive radio techniques under practical imperfections: A survey", IEEE Communications Surveys\& Tutorials, vol. 17, no. 4, pp. 1858-1884, 2015.

12. Y. C. Liang, Y. Zeng, E. C. Y. Peh and A. T. Hoang, "Sensing throughput trade-off for cognitive radio networks", In Proc of IEEE international conference on communications (ICC 2007), Glasgow, pp. 5330-5335, 2007.

13. Y. C. Liang, Y. Zeng, E. C. Y. Peh and A. T. Hoang, "Sensingthroughput trade-off for cognitive radio networks", IEEE Transactions on Wireless Communications, vol. 7, no. 4, pp.13261337, 2008.

14. S. Stotas and A. Nallanathan, "On the throughput maximization of spectrum sharing cognitive radio networks", In Proc. of IEEE global telecommunications conference (GLOBECOM2010), Miami, FL, 610 Dec 2010, pp. 1-5, 2010.

15. S. Pandit and G. Singh, "Throughput maximization with reduced data loss rate in cognitive radio networks", Telecommunication System, vol. 57, no. 2, pp. 209-215, 2014

16. J. Yang and H. Zhao, "Enhanced throughput of cognitive radio networks by improved spectrum prediction", IEEE Communication Letter 19 (10), pp.1338-1341, 2015.

17. P. Thakur et al., "Performance analysis of cognitive radio networks using channel-prediction-probabilities and improved frame structure", Digital Communication and Networks 4(2018): pp. 287-295, 2018.

\section{AUTHORS PROFILE}

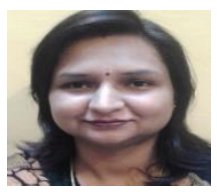

Neetu Goyal received the B.E. degree from Dr. Bhim Rao Ambedker University, Agra, India in 2002 and M.Tech. degree from Govind Ballabh Pant University of Agriculture and Technology, Pantnagar, India in 2005. She is pursuing her Ph.D. from Govind Ballabh Pant University of Agriculture and Technology, Pantnagar, India. Her field of interest includes Cognitive radio, signal processing and Neural network systems.

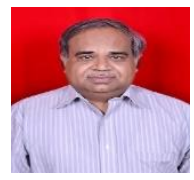

Sanjay Mathur received BE degree from Madan Mohan Malaviya Engineering College (Presently MMM University of Technology), Gorakhpur, India in 1985, ME degree from the University of Roorkee (presently IIT Roorkee), India in 1996, and Ph.D. from Govind Ballabh Pant University of Agriculture and Technology, Pantnagar, India in 2009. Currently, he is a Professor in the Department of Electronics and Communication Engineering, Govind Ballabh Pant University of Agriculture and Technology, Pantnagar. His research interests include communication systems, signal processing, neural networks and smart antennas. He is a Fellow of Institution of Engineers (India) and Life member of Indian Society of Technical Education. 